$62^{\text {ème }}$ Congrès de la SFCO, 03019 (2014)

DOI: $10.1051 /$ sfco/20146203019

(C) Owned by the authors, published by EDP Sciences, 2014

\title{
POSTER
}

\section{La maladie de Riga-Fede : observation de deux cas.}

\author{
Lafon $\mathrm{A}^{1,2}$, Ahossi $\mathbf{V}^{1}$, Grammatica $\mathrm{M}^{1}$, Zwetyenga ${ }^{3}$, Petit-Jacquin MA ${ }^{4}$, Lefevre $\mathrm{B}^{2}$, Vabres $\mathrm{P}^{4}$ \\ 1 Service d'Odontologie, Hôpital général, 3 rue Faubourg Raines, Dijon, FRANCE \\ 2 Unité fonctionnelle de Chirurgie buccale, Pôle d'Odontologie, Hôpital Maison blanche, 45 rue Cognacq-Jay, Reims, FRANCE \\ 3 Service de Chirurgie Maxillo-faciale, Hôpital du bocage, 1 boulevard Jeanne d'Arc, Dijon, FRANCE \\ 4 Service de Dermatologie, Hôpital du bocage, 1 boulevard Jeanne d'Arc, Dijon, FRANCE
}

La maladie de Riga-Fede (RF) est en rapport avec la survenue des dents néonatales et l'émergence des incisives lactéales inférieures. La maladie de RF est une lésion dentaire d'origine traumatique retrouvée sur la langue dans $60 \%$ des cas, sur la lèvre inférieure $(20 \%)$, le palais et la gencive vestibulaire maxillaire (Joseph 2010). Elle est retrouvée le plus souvent chez le nourrisson (âgé de quelques semaines à un an) et est provoquée par des mouvements répétitifs d'avant en arrière de la langue sur les incisives inférieures. (Slayton 2000). Elle est unique bien que des lésions multiples ont déjà été décrites (Joseph 2010). Elle touche les deux sexes de façon identique. Deux cas sont rapportés.

Pour le premier cas, il s'agit d'une ulcération de $10 \mathrm{~mm}$ de diamètre présente sur la face ventrale de la langue d'un nourrisson de 9 mois. Le motif de la consultation est une inquiétude des parents devant cette lésion. L'ulcération est circulaire sur un fond induré entourée d'une zone hyperkératosique, elle est en rapport direct avec les dents lactéales 71 et 81. La palpation est indurée et n'induit pas de réflexe opposant de l'enfant. Le nourrisson n'est pas gêné pour l'alimentation et les parents n'indiquent pas de gêne particulière exprimée par l'enfant. L'élimination des bords mutilants a fait disparaitre l'ulcération au bout de quelques jours.
Le cas suivant décrit une érosion de $5 \mathrm{~mm}$ de diamètre présente sur la face ventrale de la langue chez un nouveau né de cinq semaines du service néonatologie. L'ulcération fait face à deux dents néo-natales, mobiles, en place de 71 et 81 . Face au risque d'inhalation et d'ingestion des dents néonatales les dents sont avulsées.

La maladie de RF doit être prise en charge au plus vite car la lésion peut induire une déformation et une mutilation de la langue pouvant entrainer une déshydratation, un déficit alimentaire ainsi qu'à l'extrême un retard de croissance (Eley 2010). Un contrôle doit être fait après 3 semaines pour s'assurer de la guérison complète de l'ulcération après avoir supprimée l'agent traumatique. La maladie de RF peut être associée avec de graves dysfonctions neurologiques telles que le syndrome de Riley-Day, de Lesh-Nyman ou de Tourette (Choi SC 2009).

LAFON Arnaud lafon.arn@gmail.com

This is an Open Access article distributed under the terms of the Creative Commons Attribution License 4.0, which permits unrestricted use, distribution, and reproduction in any medium, provided the original work is properly cited. 